\title{
DIVISION RINGS AND $V$-DOMAINS
}

\author{
RICHARD RESCO
}

\begin{abstract}
Let $D$ be a division ring with center $k$ and let $k(x)$ denote the field of rational functions over $k$. A square matrix $\tau \in M_{n}(D)$ is said to be totally transcendental over $k$ if the evaluation map $\varepsilon: k[x] \rightarrow M_{n}(D), \varepsilon(f)=f(\tau)$, can be extended to $k(x)$. In this note it is shown that the tensor product $D \otimes_{k} k(x)$ is a $V$-domain which has, up to isomorphism, a unique simple module iff any two totally transcendental matrices of the same order over $D$ are similar. The result applies to the class of existentially closed division algebras and gives a partial solution to a problem posed by Cozzens and Faith.
\end{abstract}

An associative ring $R$ is called a right $V$-ring if every simple right $R$-module is injective. While it is easy to see that any prime Goldie $V$-ring is necessarily simple [5, Lemma 5.15], the first examples of noetherian $V$-domains which are not artinian were constructed by Cozzens [4]. Now, if $D$ is a division ring with center $k$ and if $k(x)$ is the field of rational functions over $k$, then an observation of Jacobson [7, p. 241] implies that the simple noetherian domain $D \otimes_{k} k(x)$ is a division ring iff the matrix ring $M_{n}(D)$ is algebraic over $k$ for every positive integer $n$. In their monograph on simple noetherian rings, Cozzens and Faith raise the question of whether this tensor product need be a $V$-domain [5, Problem (13), p. 116]. Although it will be shown that the general question has a negative answer, the basic result of this paper characterizes those division rings for which $D \otimes_{k} k(x)$ is a $V$-domain possessing, up to isomorphism, a unique simple module. Combined with a theorem of Cohn [2], the result leads to a corollary which closely parallels Cozzens' original example of the ring of differential operators over a universal differential field [4].

Throughout the paper $k$ will be a commutative field and $k(x)$ will denote the field of rational functions over $k$. Tensor products, when not specifically marked, are taken relative to $k$. Modules are right modules, and accordingly module maps are written on the left. The author would like to thank Robert Guralnick for several stimulating conversations, and to thank George M. Bergman for pointing out Example 2.

A necessary preliminary to the proof of the main theorem is the description of the finite length $(D \otimes k(x))$-modules given below. The key ingredient in this description is the notion of a transcendental matrix introduced in [2]. In order to avoid confusion with the traditional meaning of the term "transcendental," however, we use a slightly different terminology here.

Received by the editors August 24, 1984 and, in revised form, January 13, 1986.

1980 Mathematics Subject Classification (1985 Revision). Primary 16A39, 16A52, 16A33.

$K e y$ words and phrases. Division algebras, $V$-rings.

This work was supported in part by a grant from the National Science Foundation. 
DEFINITION 1 (COHN). Let $D$ be a division algebra over the field $k$ and let $\tau \in M_{n}(D)$ be an $n \times n$ matrix over $D$. We say that $\tau$ is totally transcendental over $k$ if for every nonzero polynomial $f(x) \in k[x]$ the evaluation $f(\tau)$ is a unit in $M_{n}(D)$.

If $\alpha$ is an arbitrary $n \times n$ matrix over $D$, then associated with $\alpha$ there is a canonical module over the polynomial ring $D[x]$, which we shall denote by $\left(D^{n}, \alpha\right)$. In detail, if one views $D^{n}$ as column $n$-space over $D$, then $\left(D^{n}, \alpha\right)$ is the right $D[x]$-module obtained by letting the elements of $D$ act by right multiplication and by letting the indeterminate $x$ act by left multiplication by $\alpha$. If $\alpha$ is totally transcendental over $k$, moreover, this action can be extended to $T=D \otimes k(x)$ and $\left(D^{n}, \alpha\right)$ is naturally a $T$-module. We now show that if $D$ is a division algebra for which $T$ is not artinian, then every $T$-module of finite length arises in this fashion.

LEMMA. Let $D$ be a division algebra over the field $k$ and let $T$ denote the tensor product $D \otimes k(x)$. If $T$ is not a division ring, then given any nonzero $T$-module $L$ of finite length, there exist a positve integer $m$ and $a \beta \in M_{m}(D)$, which is totally transcendental over $k$, such that $L$ is isomorphic to $\left(D^{m}, \beta\right)$.

PROOF. We first show that $L_{D}$, the $D$-module obtained by restriction of scalars, is finite demensional over $D$. Since $T$ is a principal left and right ideal domain, $L$ is a direct sum of cyclic torsion modules. Thus, for our purpose, it is enough to consider the case $L=T / J$, where $J$ is a nonzero right ideal of $T$. One can view $T$ as the localization of $R=D[x]$ at the central multiplicative subset $S=k[x]-\{0\}$, so if $I=J \cap R$ and $K=R / I$, then $L \approx K \otimes_{R} T$. Now if $I=0$, this would make $L \approx T$, but since $T$ is not a division ring it has infinite length. So $I \neq 0$, whence $K_{D}$ is finite dimensional by the right division algorithm. Now, if $s \in S$, the action of $s$ on $K$ induces a $D$-endomorphism $p_{s}$ of $K$. Inasmuch as $K$ is $S$-torsion free by construction, $p_{s}$ is injective. But $D$ is a division ring, so $p_{s}$ is also surjective and $K$ is $S$-divisible. It follows that $K$ and $L$ are isomorphic as $R$-modules, all the more so as $D$-modules.

To complete the proof it suffices to take a $D$-basis of $L_{D}$ and to let $\beta$ be the matrix of $p_{x}$ with respect to that basis.

Before proceeding to the proof of the theorem, we apply the lemma to show that if $D$ is the most familiar example of a nonalgebraic division ring, then $D \otimes k(x)$ is not a $V$-domain.

EXAMPLE 1. Let $k$ be a field of characteristic zero, let $A_{1}$ denote the first Weyl algebra over $k$, and let $D_{1}$ be the quotient division ring of $A_{1}$. As is well known, the center of $D_{1}$ is $k$, and as any $d \in D_{1}-k$ is transcendental over $k$ (for an easy proof, see [9, Theorem 4.ii]), $T=D_{1} \otimes k(x)$ is not a division ring. Let $p$ and $q$ be the canonical generators of $A_{1}$ and form the $T$-module $M=\left(D_{1}, q p\right)$, which is obviously simple. If $M$ is injective, then it is divisible. In particular, there exists an $a \in D_{1}$ such that $a \cdot(1 \otimes x-q p \otimes 1)=1$, that is, $q p a-a q p=1$. We claim, however, that this equation has no solution in $D_{1}$. To verify the claim, it is convenient to introduce a larger division algebra which contains an isomorphic image of $D_{1}$. Let $K=k(t)$ be a rational function field over $k$, let $\sigma: K \rightarrow K$ be the $k$-automorphism $\sigma(t)=t+1$, and let $L=K((s: \sigma))$ be the division ring of left skew Laurent series over $K$. In $L$ the elements $s$ and $t$ satisfy $s\left(t s^{-1}\right)-\left(t s^{-1}\right) s=1$, hence there exists a $k$-algebra embedding $f: D_{1} \rightarrow L$ with $f(p)=s$ and $f(q)=t s^{-1}$. If $[q p, a]=1$ 
has a solution in $D_{1}$, then $[t, b]=1$ has a solution in $L$. Writing $b=\sum_{i=-n}^{\infty} r_{i} s^{i}$ $\left(r_{i} \in K\right)$ and equating coefficients in the relation $t b-b t=1$, we obtain $r_{i}=0$ for all $i \neq 0$ and $t r_{0}-r_{0} t=1$. Since $K$ is commutative, this is impossible.

To make the statement of the theorem and the remarks which follow it less awkward, we need another definition.

DEFINITION 2. Let $D$ be a division ring with center $k$. We say that $D$ has the conjugacy property if given any positive integer $n$ and given any two matrices $\alpha, \beta \in M_{n}(D)$, both of which are totally transcendental over $k$, there exists a unit $\gamma \in M_{n}(D)$ with $\gamma \alpha=\beta \gamma$.

THEOREM. Let $D$ be a division ring with center the field $k$, and let $T$ denote the tensor product $D \otimes k(x)$. Then the following are equivalent:

(i) $T$ is a $V$-domain which has, up to isomorphism, a unique simple module;

(ii) $D$ has the conjugacy property.

Proof. First note that if $T$ is a division ring, then (i) is obvious and (ii) is vacuous by the observation of Jacobson noted earlier, so their equivalence is trivial. For the remainder of the proof, therefore, we assume that $T$ is not a division ring.

Suppose that (i) holds and let $M$ be a simple $T$-module. Inasmuch as $M$ is injective and inasmuch as any other simple $T$-module is isomorphic to $M$, an induction on composition length shows that any $T$-module of finite length $l$ is isomorphic to the direct sum $M^{l}$. Now let $\alpha, \beta \in M_{n}(D)$ be totally transcendental over $k$, and form the $T$-modules $\left(D^{n}, \alpha\right),\left(D^{n}, \beta\right)$. If $l\left(L_{T}\right)$ denotes the length of a finitely generated torsion $T$-module $L$, then the relation $\operatorname{dim}\left(L_{D}\right)=l\left(L_{T}\right) \cdot \operatorname{dim}\left(M_{D}\right)$ (and the fact that the latter dimension is finite, since $T$ is not a division ring) implies that $\left(D^{n}, \alpha\right)$ and $\left(D^{n}, \beta\right)$ have the same length over $T$, hence are isomorphic. If $g:\left(D^{n}, \alpha\right) \rightarrow\left(D^{n}, \beta\right)$ is a $T$-isomorphism, then a standard argument shows that $g$ is given by left multiplication by an invertible matrix $\gamma \in M_{n}(D)$, which satisfies $\gamma \alpha=\beta \gamma$.

To prove the converse, we first show that if $D$ has the conjugacy property, then any two simple $T$-modules are isomorphic. So suppose that $M$ and $N$ are simple. Since $T$ is not a division ring, there exists positive integers $n, m$ and totally transcendental matrices $\alpha \in M_{n}(D), \beta \in M_{m}(D)$ such that $M \approx\left(D^{n}, \alpha\right)$ and $N \approx\left(D^{m}, \beta\right)$. Let $\bigoplus_{m} \alpha$ (respectively, $\bigoplus_{n} \beta$ ) denote the block-diagonal sum of $m$ copies of $\alpha(n$ copies of $\beta)$. Easily, both $\bigoplus_{m} \alpha$ and $\bigoplus_{n} \beta$ are totally transcendental over $k$, and being of the same order over $D$, they are conjugate. It follows that the $T$-modules $M^{m}$ and $N^{n}$ are isomorphic, whence $\operatorname{Hom}_{T}(M, N) \neq 0$. As $M$ and $N$ are simple, this forces $M$ to be isomorphic to $N$.

It remains to show that the essentially unique simple $T$-module is injective. If $M \approx\left(D^{n}, \alpha\right)$ is simple and if $L \approx\left(D^{m}, \beta\right)$ has finite length $l$, then the relation between dimension and length noted in the second paragraph of the proof gives $m=\ln$. Since $\beta$ is conjugate to $\bigoplus_{l} \alpha, L$ is isomorphic to the semisimple module $M^{l}$. It readily follows that any torsion $T$-module is semisimple. In particular, if $E(M)$ is an injective hull of $M$, then $E(M)$ is torsion and we must have $M \approx E(M)$.

A class of division algebras all of whose members satisfy the conjugacy property nonvacuously is the class of existentially closed division algebras. For the definition and basic properties of these division rings, we refer the reader to $[\mathbf{3}, \S 6.2 ; \mathbf{6}]$. 
COROLLARY. If $k$ is a field and if $E$ is an existentially closed division algebra over $k$, then $E \otimes k(x)$ is a nonartinian $V$-domain which possesses, up to isomorphism, a unique simple module.

Proof. By $[3$, p. 137] the center of $E$ is $k$. Since $E$ contains commutative subfields of arbitrary finite transcendence degree over $k$ [6, Theorem 14.10] $E \otimes k(x)$ cannot be a division ring. Finally, [2, pp. 161-162] shows that $E$ has the conjugacy property.

A natural question raised by the corollary is whether its converse is true. While the analogous problem for a ring of differential operators over a commutative field is apparently still open (compare [5, Theorem 5.21] with [8, §I.5]), G. M. Bergman [1] has resolved the former question by constructing a counterexample. We conclude this note by presenting Bergman's example.

EXAMPLE 2 (BERGMAN). Let $k$ be the field of rational numbers, and let $H$ be the quaternion algebra over $k$, with canonical generators $i$ and $j$ satisfying $j i=$ $-i j, i^{2}=-1=j^{2}$. By $[3$, Theorem 6.2.2] $H$ can be embedded in an existentially closed division ring $E$. If $D$ is the centralizer of $H$ in $E$ and if $C$ is the center of $D$, then we claim that: (i) $D$ is not algebraic over $C$; (ii) $D$ has the conjugacy property; (iii) $D$ is not existentially closed.

(i) Let $A_{1}$ be the Weyl algebra over $k$, and for any $k$-algebra $B$ let $A_{1}(B)$ denote the tensor product $A_{1} \otimes B$. If $k\langle u, v\rangle$ is a free algebra of rank two over $k$, then the system of equations $[u, v]=1,[u, i]=[u, j]=[v, i]=[v, j]=0$ (viewed as polynomials in the free product $E \sqcup_{k} k\langle u, v\rangle$ ) has a solution in the quotient division ring of $A_{1}(E)$. Since $E$ is existentially closed over $k$, the system has a solution in $E$, but the last four equations show that any such solution lies in $D$. If $d_{1}, d_{2} \in D$ are explicit solutions to the system, then there is a $k$-algebra embedding of $A_{1}(C)$ into $D$, whose image is the $C$-subalgebra of $D$ generated by $d_{1}$ and $d_{2}$. Thus, $d_{1}$ is transcendental over $C$.

(ii) We make some minor adjustments to the argument used in the proof of [2, Theorem 4.2]. Let $\alpha, \beta \in M_{n}(D)$ be totally transcendental over $C$, and let $F=E((t))$ be the ring of Laurent series over $E$. Let $H(t)$ denote the $k$-subalgebra of $M_{n}(F)$ generated by $H$ and the field $k(t)$, and $H(\alpha)$ that generated by $H$ and $k(\alpha)$. Inasmuch as $H$ is finite dimensional over $k, H \otimes k(x)$ is a division ring. It easily follows that $H(t)$ and $H(\alpha)$ are division subalgebras of $M_{n}(F)$ and that there exists an isomorphism $f: H(t) \rightarrow H(\alpha)$ with $f(t)=\alpha$ and $f(h)=h$ for all $h \in H$. Similarly, if $H(\beta)$ is the $k$-subalgebra of $M_{n}(F)$ generated by $H$ and $k(\beta)$, then $H(\beta)$ is a division algebra and there exists an isomorphism $g: H(t) \rightarrow H(\beta)$ with $g(t)=\beta$ and $g(h)=h$ for all $h \in H$. If $H(t, \alpha)$ (respectively, $H(t, \beta)$ ) is the subalgebra of $M_{n}(F)$ generated by $H(t)$ and $H(\alpha)(H(t)$ and $H(\beta))$, then each of $H(t, \alpha)$ and $H(t, \beta)$ is isomorphic to $H \otimes k(x, y)$, hence each is a division ring. By [2, Lemma 4.1] and the remark which immediately follows it, there exist a division ring $G \supset F$ and an invertible matrix $\gamma \in M_{n}(G)$ such that $\gamma a \gamma^{-1}=g f^{-1}(a)$ for all $a \in H(\alpha)$. The system of equations deducible from the matrix relations $\mu \alpha=\beta \mu, \mu \nu=1,[\mu, i]=0,[\mu, j]=0$ is thus consistent over $E$, and we can take $\gamma$ to be an element of $M_{n}(D)$.

(iii) The single-variable equation $u^{2}=-1$ is obviously consistent over $D$. If there exists a $d$ in $D$, which is a solution to this equation, then $K=k[i, d]$ is a finite-dimensional commutative subalgebra of $E$, hence a field. Since the given 
equation can have at most two solutions in $K$, we get $i= \pm d \in D$. This implies that $i$ commutes with $j$, which is absurd.

\section{REFERENCES}

1. G. M. Bergman, Private communication, December 1981.

2. P. M. Cohn, The similarity reduction of matrices over a skew field, Math. Z. 132 (1973), 151-163.

3. _ Skew field constructions, London Math. Soc. Lecture Notes Series, vol. 27, Cambridge Univ. Press, Cambridge, 1977.

4. J. H. Cozzens, Homological properties of the ring of differential polymomials, Bull. Amer. Math. Soc. 76 (1970), 75-79.

5. J. Cozzens and C. Faith, Simple Noetherian rings, Cambridge Univ. Press, Cambridge, 1975.

6. J. Hirschfeld and W. H. Wheeler, Forcing, arithmetic, division rings, Lecture Notes in Math., vol. 454, Springer-Verlag, Berlin and New York, 1975.

7. N. Jacobson, The structure of rings, Amer. Math. Soc. Colloq. Publ., vol. 37, Amer. Math. Soc., Providence, R.I., 1964.

8. E. R. Kolchin, Galois theory of differential fields, Amer. J. Math. 75 (1953), 753-824.

9. R. Resco, L. W. Small, and A. R. Wadsworth, Tensor products of division rings and finite generation of subfields, Proc. Amer. Math. Soc. 77 (1979), 7-10.

Department of MATHEMATICS, UNiversity of OKLAHOMA, NORMAN, OKLAHOMA 73019 